Original article

\title{
Knowledge of gestational diabetes mellitus among pregnant women in a semiurban hospital - A cross -sectional study
}

\author{
Karthiga Prabhu J*, Surya Deepthi Kondamuri, Sunita Samal, Maitrayee Sen \\ Department of Obstetrics and Gynaecology, SRM Medical College Hospital and Research Centre, SRM Institute of Science and Technology, Kattankulathur, Chengelpet, \\ 603203, Tamilnadu, India
}

\section{A R T I C L E I N F O}

\section{Keywords:}

GDM

Prevention

Diabetes mellitus

Screening

Pregnant women

Knowledge

\begin{abstract}
A B S T R A C T
Background: Prevalence of gestational diabetes is on the rise throughout the world, particularly in south east Asia. Gestational diabetes affects both present and future generations. The present study was conducted to study the knowledge about GDM, among antenatal women in a tertiary care hospital located in semiurban area.

Methodology: In this cross-sectional questionnaire based study, a prevalidated questionarre with 18 questions regarding general awareness and knowledge about risk factors of GDM, screening of GDM, treatment modalities, immediate and long term consequences of GDM were used. Participants were divided into 3 groups based on knowledge score - good (score $>12$ ), fair (score 7-12) and poor (score $\leq 6$ ) knowledge.

Results: Among the 200 study participants, only 92 (46\%) knew that diabetes can occur for the first time during pregnancy. Good knowledge about GDM was present in 103 (51.5\%), fair knowledge in 68 (34\%) and poor knowledge in 29 (14.5\%). Among the knowledge about risk factors of GDM, family history of diabetes was considered as a major risk factor $(84.5 \%)$ followed by obesity $(53.5 \%)$. Only $34.5 \%$ of patients were aware that GDM screening should be done for all antenatal women. Knowledge about fetal and neonatal complications was seen in only $30-50 \%$ of participants. Education and family history of diabetes were found to be significantly associated with better knowledge score $(\mathrm{p}=0.0002, \mathrm{p}=0.03$ respectively).

Conclusion: Awareness about effect of GDM on the neonate and the mother should be created to improve GDM screening especially in non graduates and those residing in rural areas. Grass root level workers should be more involved in spreading awareness to all antenatal mothers from rural areas.
\end{abstract}

\section{Introduction}

Gestational diabetes mellitus (GDM) is a defined as glucose intolerance of varying severity that begins or is first recognized during pregnancy. Globally the prevalence of gestational diabetes is on the rise particularly in south east Asia. Globally the prevalence of GDM varies between $1 \%$ and $28 \%$ depending on screening methods and population characteristics. ${ }^{1}$ In some developing countries, especially in India the prevalence rates are higher and ranges from 3.8\% in Kashmir to $17.9 \%$ in Tamil nadu. ${ }^{2,3}$ Increase in prevalence may be due to advanced maternal age, increased BMI and ethnicity. Frequency of gestational diabetes parallels increase in type 2 Diabetes mellitus in a particular population. ${ }^{2}$ The common risk factors of GDM include previous obstetric history of GDM, history of large for gestational age babies, abortions and unexplained stillbirths in the previous pregnancies and family history of both GDM and Type 2DM. Inter pregnancy weight gain is also considered as one of the most common modifiable risk factor for GDM.

Abnormal levels of blood glucose in pregnant women can affect immediate and long term health of both mother and baby. GDM affects two generations both the present and the next generation. GDM is associated with increased risk of maternal and neonatal complications like preeclampsia, macrosomia, shoulder dystocia, birth injuries, increased risk of caesarean delivery, hypoglycemia, neonatal jaundice respiratory distress syndrome, polycythemia and hypocalcemia in newborn. Both GDM mother and her offspring are at risk of Type 2 Diabetes and obesity in future. There is a $50 \%$ risk of gestational diabetic mothers to develop overt diabetes within 20 years. . $^{4,5}$

Thus, GDM screening will provide a perfect window of opportunity for the prevention of DM in two generations. Knowledge about GDM among antenatal women will facilitate implementation of healthy lifestyle, better healthcare seeking behaviour and thus prevention and early diagnosis of disease.

\footnotetext{
* Corresponding author.

E-mail address: j.karthigaprabhu@gmail.com (K. Prabhu J).
} 
Hence, the objective of our study was to determine the knowledge and awareness of GDM, risk factors, screening and immediate and future complications of GDM among pregnant women attending our antenatal clinic.

\section{Methodology}

\subsection{Study design, setting, and subjects}

This cross-sectional questionnaire based study was conducted in the department of Obstetrics and Gynaecology over a period of 3 months. The study population included pregnant women who had their first visit to the Antenatal clinic of SRM Medical College Hospital and Research Centre, a tertiary care hospital located in semiurban area, and who were willing to participate in the study. Baseline characteristics including sociodemographic data, educational level, occupation, parity, association with medical field, familial history of diabetes, history of GDM in previous pregnancy were noted. A prevalidated questionarre with 18 questions regarding general awareness and knowledge about risk factors of GDM, screening of GDM, treatment modalities, immediate and long term consequences of GDM were used. The answer options provided were yes/no. Each correct response was given a score of 1 and each women was scored out of total 18 . Scoring was given as below $\leq 6$ - poor knowledge, 7- 12 - fair knowledge and $>12$ - good knowledge. The source from where the antenatal women got knowledge regarding GDM was also noted.

Ethical approval of studies and Informed consent: This study was conducted with the approval of the institutional ethics committee (2433/IEC/2021) of SRM Medical College Hospital and Research Centre and informed consent has been obtained from all study participants.

Statistical Analysis: The data were entered in Microsoft excel and analysed by using statistical software SPSS (Statistical package for Social Sciences) version 19.0. Descriptive statistics such as frequency, percentage for qualitative variables and mean, standard deviation for quantitative variables were used to describe the data. Inferential statistics such as Chisquare test was used to compare the proportions and ANOVA was used to find the difference in the means between the groups. $P$ value less than 0.05 was considered significant.

\section{Results}

During the study period 200 participants were included in the study. Majority (95\%) of them were between 20 and 34 years. Majority (72\%) of participants were from rural and semiurban areas. Among the study participants $64.5 \%$ were housewives and $58 \%$ were graduates. Familial history of diabetes mellitus was present in $40.5 \%$ and $14.5 \%$ had gestational diabetes in the previous pregnancy (Table 1). Among the participants, only $92(46 \%)$ knew that diabetes can occur for the first time during pregnancy.

On overall scoring, good knowledge about GDM was present in 103 (51.5\%), fair knowledge in 68 (34\%) and poor knowledge in 29 (14.5\%) among the study participants. On analysing the knowledge about risk factors of GDM, family history of diabetes was considered as a major risk factor (84.5\%) followed by obesity (53.5\%). About $72 \%$ of patients were aware that screening is done by giving glucose load and $74 \%$ of patients were of the opinion that they should do screening in fasting status. Only $34.5 \%$ of patients were aware that GTT should be done for all antenatal women. Nearly $70 \%$ of patients were aware about the various treatment modalities available for management of GDM. Knowledge about fetal and neonatal complications was seen in only $30-50 \%$ of participants. Knowledge about future risk of diabetes mellitus in mother and offspring was present in $67 \%$ and $47 \%$ respectively (Table 2 ).

Among the participants from urban area, 57\% had good knowledge
Table 1

Demographic data

\begin{tabular}{lll}
\hline Baseline characteristics & $\mathrm{N}=200$ & $\%$ \\
\hline Age & \multicolumn{1}{l}{} \\
$<19$ years & 190 & 3 \\
$20-34$ yrs & 4 & 95 \\
$>35$ yrs & & 2 \\
Residence & 56 & \\
Urban & 82 & 28 \\
Semiurban & 62 & 41 \\
Rural & & 31 \\
Employment & 129 & \\
Housewife & 4 & 64.5 \\
Employed in medical field & 67 & 2 \\
Employed in other fields & & 33.5 \\
Education & 2 & \\
Primary school & 82 & 1 \\
Secondary school & 116 & 41 \\
Graduate & 23 & 58 \\
Relatives working in medical field & 81 & 11.5 \\
Family history of diabetes & 29 & 40.5 \\
Previous history of GDM & & 14.5 \\
\hline
\end{tabular}

Table 2

Knowledge of antenatal women on various aspects of gestational diabetes mellitus.

\begin{tabular}{|c|c|c|c|}
\hline S.no & $\begin{array}{l}\text { Parameters } \\
\text { Knowledge about GDM risk factors }\end{array}$ & No. & $\%$ \\
\hline 1. & Family history of Diabetes Mellitus & 149 & 84.5 \\
\hline 2. & Obesity & 107 & 53.5 \\
\hline 3. & GDM in previous pregnancies & 91 & 45.5 \\
\hline 4. & $\begin{array}{l}\text { Rapid weight gain during pregnancy } \\
\text { Knowledge about GDM screening }\end{array}$ & 103 & 51.5 \\
\hline 5. & Glucose load & 144 & 72 \\
\hline 6. & Screening for all antenatal women & 69 & 34.5 \\
\hline 7. & $\begin{array}{l}\text { Fasting status } \\
\text { Knowledge about treatment options }\end{array}$ & 148 & 74 \\
\hline 8. & Diet and exercise & 138 & 69 \\
\hline 9. & Insulin & 132 & 66 \\
\hline 10. & $\begin{array}{l}\text { Oral medications } \\
\text { Knowledge about immediate complications }\end{array}$ & 139 & 69.5 \\
\hline 11. & Abortions & 64 & 32 \\
\hline 12. & Congenital anomalies & 86 & 43 \\
\hline 13. & Big baby & 102 & 51 \\
\hline 14. & Preterm delivery & 70 & 35 \\
\hline 15. & $\begin{array}{l}\text { Neonatal problems } \\
\text { Knowledge about future course: }\end{array}$ & 85 & 42.5 \\
\hline 16. & Disappears after delivery & 99 & 49.5 \\
\hline 17. & Future risk of diabetes for the mother & 134 & 67 \\
\hline 18. & Future risk of diabetes for newborn & 94 & 47 \\
\hline
\end{tabular}

about GDM when compared to $43 \%$ in rural area and $46.7 \%$ in semiurban area. Among graduates $63.7 \%$ had good knowledge, $26 . \%$ had fair knowledge and $10.3 \%$ had poor knowledge. In contrast in individuals with secondary school education $45.1 \%$ had fair knowledge and $34.14 \%$ had good knowledge which was statistically significant $(\mathrm{p}=0.0002)$. All 4 participants who were associated with medical field had good knowledge about GDM. Among participants with familial history of DM $60.4 \%$ had good knowledge when compared to $41.8 \%$ in those without familial history ( $\mathrm{p}=0.03$ ) (Table 3 ).

Participants from urban area had statistically significant knowledge score on GDM treatment modalities and fetal and neonatal complications when compared to rural and semiurban areas $(\mathrm{p}=0.015, \mathrm{p}=$ 0.028 respectively).Except knowledge about GDM screening, knowledge score for all other factors was significantly more in graduates compared to participants with primary and secondary education. Though knowledge score was better in employed women than in unemployed women, only knowledge score about future course was significantly more in 
Table 3

Comparison of knowledge score based on Residential area, Education, Occupation, Familial history and Previous history of GDM.

\begin{tabular}{llllll}
\hline Parameters & Score & & & Chi square & p \\
\hline & Poor & Fair & Good & & \\
Residence & & & & 8.654 & 0.07 \\
Urban (56) & 6 & 12 & 38 & & \\
Rural (82) & 12 & 34 & 36 & & \\
Semiurban (62) & 11 & 22 & 29 & & 0.002 \\
Education & & & & 17.46 & \\
Graduate (116) & 12 & 30 & 74 & & \\
Secondary school (82) & 17 & 37 & 28 & & \\
Primary school (2) & 0 & 1 & 1 & & \\
Occupation & & & & 4.211 & \\
Housewife (129) & 20 & 43 & 66 & & \\
Employed in medical field (4) & 0 & 0 & 4 & & \\
Employed in other fields (67) & 9 & 25 & 33 & & \\
Family history of DM & & & & 7.006 & \\
Yes (81) & 6 & 26 & 49 & & \\
No (119) & 23 & 42 & 54 & & \\
Previous history of GDM & & & & 1.482 & \\
Yes (27) & 3 & 12 & 12 & & \\
No (173) & 26 & 56 & 91 & & \\
\hline
\end{tabular}

participants employed in medical field when compared to others ( $\mathrm{p}=$ 0.009). (Table 4). In our study major source of information regarding GDM awareness is from family members and friends $(69 \%, 62 \%)$ (Fig. 1).

\section{Discussion}

Prevalence of Type 2 Diabetes in the world is on the rise particularly in South -East Asia. ${ }^{6}$ This increase parallels with the increase in GDM. Identifying and managing GDM early may prevent the development of future Diabetes. For diagnosing GDM early, antenatal women should be aware about the riskfactors, screening methods and complications. To prevent and minimize maternal and fetal morbidity associated with GDM, Ministry of Health and Family Welfare has released a national guideline for providing universal screening and management of GDM as part of the essential antenatal package.

In our study only $46 \%$ were aware that Diabetes can occur for the first time in pregnancy. In a study by Pierce et al. (2017) in Samoa, 58\% women were aware that diabetes can occur for the first time during pregnancy. ${ }^{7}$ In a study from primary health centre in south India by Shriram et al. $68.7 \%$ were aware that diabetes can occur during pregnancy. ${ }^{8}$ This variation in awareness may be due to difference in health education among different sectors of people. Though Tamilnadu government has implemented universal screening for GDM by means of a single blood glucose test $2 \mathrm{~h}$ post $75 \mathrm{~g}$ of glucose in all the three trimesters, it has not reached different sections of the society. In primary health centres, the part played by the Anganwadi workers and other ground level workers may be the reason for better awareness among participants in the study by Shriram et al. ${ }^{8}$ In contrast in our study done in a semiurban area with participants from different localities, awareness about GDM was low, showing that health education has not reached all sectors of people. Hence, innovative ideas should be framed to

Table 4

Difference in Knowledge score based on Residential area, Education and occupation.

\begin{tabular}{|c|c|c|c|c|c|}
\hline \multirow[t]{2}{*}{ Knowledge Parameter (score) } & \multicolumn{3}{|l|}{ Area } & \multirow[t]{2}{*}{ ANOVA } & \multirow[t]{2}{*}{$\mathrm{P}$ value } \\
\hline & Urban & Rural & Semiurban & & \\
\hline Risk factor (4) & $2.47 \pm 1.28$ & $2.06 \pm 1.30$ & $2.23 \pm 1.20$ & 1.588 & 0.207 \\
\hline GDM Screening (3) & $1.84 \pm 0.88$ & $1.77 \pm 1.03$ & $1.81 \pm 0.95$ & 0.076 & 0.926 \\
\hline Treatment (3) & $2.35 \pm 0.86$ & $1.81 \pm 1.19$ & $2.01 \pm 0.99$ & 4.286 & 0.015 \\
\hline Fetal \& neonatal Complication (5) & $2.46 \pm 1.34$ & $2.02 \pm 1.69$ & $1.75 \pm 1.48$ & 3.625 & 0.028 \\
\hline Future course of GDM (3) & $1.74 \pm 0.90$ & $1.71 \pm 0.93$ & $1.51 \pm 0.99$ & 1.276 & 0.281 \\
\hline \multirow[t]{2}{*}{ Knowledge Parameter (score) } & \multicolumn{3}{|c|}{ Education } & ANOVA & $P$ value \\
\hline & Graduate & Secondary school & Primary school & & \\
\hline Risk factors (4) & $2.54 \pm 1.20$ & $1.84 \pm 1.24$ & $2.00 \pm 1.41$ & 8.028 & 0.0001 \\
\hline GDM screening (3) & $1.92 \pm 0.90$ & $1.65 \pm 1.02$ & $2.00 \pm 0.00$ & 2.078 & 0.128 \\
\hline Treatment modalities (3) & $2.25 \pm 0.99$ & $1.76 \pm 1.06$ & $2.00 \pm 0.00$ & 5.692 & 0.004 \\
\hline Fetal and neonatal Complications (5) & $2.46 \pm 1.55$ & $1.45 \pm 1.32$ & $1.50 \pm 0.71$ & 11.597 & 0.0001 \\
\hline Future Course (3) & $1.76 \pm 0.97$ & $1.49 \pm 0.88$ & $0.50 \pm 0.71$ & 3.503 & 0.032 \\
\hline \multirow[t]{2}{*}{ Knowledge parameters (score) } & \multicolumn{3}{|c|}{ Occupation } & ANOVA & $P$ value \\
\hline & Employed in medical fields & Unemployed & Employed in other fields & & \\
\hline Risk factors (4) & $3,00 \pm 0.82$ & $2.19 \pm 1.28$ & $2,31 \pm 1.25$ & 0.922 & 0.399 \\
\hline GDM Screening (3) & $2.25 \pm 0.50$ & $1.81 \pm 0.97$ & $1.79 \pm 0.95$ & 0.438 & 0.646 \\
\hline Treatment modalities (3) & $2.75 \pm 0.50$ & $2.00 \pm 1.06$ & $2.09 \pm 1.01$ & 1.106 & 0.333 \\
\hline Fetal and Neonatal Complications (5) & $2.25 \pm 1.50$ & $1.93 \pm 1.56$ & $2.22 \pm 1.49$ & 0.850 & 0.429 \\
\hline Future course of GDM (3) & $2.75 \pm 0.50$ & $1.71 \pm 0.94$ & $1.43 \pm 0.92$ & 4.839 & 0.009 \\
\hline
\end{tabular}

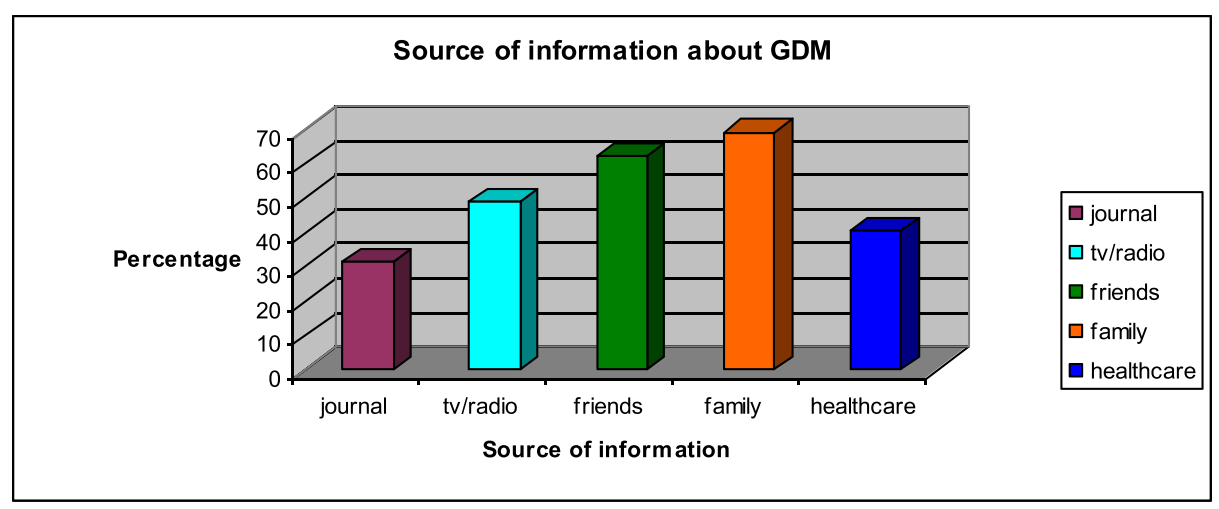

Fig. 1. Source of information about GDM. 
promote health education to reach all societies of people.

Majority of our study participants were between 19 and 34 years. Nearly $70 \%$ of the participants were from semiurban and rural areas. $64.5 \%$ of participants were housewives and $58 \%$ were graduates. In contrast in a study from Karnataka majority of women had only primary education (61\%) and most of the women were working (54.8\%) and residing in slum areas $(43.2 \%){ }^{9}$

In our study $51.5 \%$ had good knowledge and only $14.5 \%$ had poor knowledge about GDM. Dhyani et al. in his study from Karnataka showed that majority of women (57.6\%) had average knowledge about the GDM while $21.8 \%$ of the women had good knowledge and $19 \%$ had poor knowledge. ${ }^{9}$ Mahalakshmi et al. ${ }^{10}$ found only $35.2 \%$ had adequate knowledge about GDM and in the study by Shreeram et al. ${ }^{8}$ only $17.5 \%$ of women had good knowledge about GDM. Similarly, in a Bangladesh study $26.3 \%$ of study participants had good knowledge about GDM. In a multiethnic cohort study by Carolan, Vietnamese and Filipino women and caucasian women scored poorly on general knowledge about GDM while Indian women scored highest across all areas of interest. ${ }^{11}$ Indians had better knowledge probably because of the initiative taken by the government to prevent non communicable diseases. National health guidelines for GDM screening and management has been formulated and is being followed.

Family history of diabetes as a risk factor was identified in $84.5 \%$ and knowledge about lifestyle modification (Diet and exercise) as a treatment modality was seen in $69 \%$. Knowledge about future risk of diabetes was seen in $67 \%$. Only $41 \%$ had knowledge about immediate and long term complications in neonates caused by GDM. Hence, health education should be focussed on improving the knowledge about fetal and neonatal complications among antenatal women. This may promote effective follow up among pregnant women for GDM screening which facilitates early diagnosis of GDM and prevention of future complications.

Educational status of the study population was found to have significant association with knowledge about GDM ( $p=0.0002)$. Good knowledge about GDM was seen in $63.7 \%$ of graduates when compared to $34.14 \%$ individuals with secondary school education which was statistically significant. Except knowledge about GDM screening, knowledge score for riskfactors, immediate and future complications of GDM were significantly more in graduates compared to participants with primary and secondary school education. Similar significant association with education was also seen in the study by Bhownik et al. Dhyani et al. and Mahalakshmi et al.. 9,10,12 This association is because of the better access of this group to health related journals and better understanding of the health problems. Periodic conducting of health camps and creating awareness in vernacular language using role plays and using mass media like television and radio may help to tackle this problem.

Among the participants from urban area, 57\% had good knowledge about GDM when compared to $43 \%$ in rural area and $46.7 \%$ in semiurban area. Participants from urban area had statistically significant knowledge score on GDM treatment modalities and fetal and neonatal complications when compared to rural and semiurban areas $(\mathrm{p}=0.015$, $\mathrm{p}=0.028$ respectively). Bhowmik et al. and Mahalakshmi et al. also reported that those who were from urban areas had significantly higher knowledge about GDM than those from rural areas. ${ }^{10,12}$ In the study by Bhavatharini et al. $49.2 \%$ of urban women and $75.6 \%$ of rural women did not know the long-term consequences of GDM to babies born to GDM women. Awareness that GDM could lead to type 2 diabetes was seen in $50.8 \%$ in urban women compared to $45 \%$ of rural women. ${ }^{13}$ Women from urban areas have better exposed to mass media like television, Radio and Internet through which they gain more health information when compared to women from rural areas.

All participants in medical field had good knowledge about GDM. Among participants with familial history of DM $60.4 \%$ had good knowledge score when compared to $41.8 \%$ without familial history ( $\mathrm{p}=$ 0.03). In contrast in the study by Dhyani et al. statistically significant association was noted between knowledge about GDM with age, educational status, religion, and occupation $(\mathrm{P}<0.050)$. However, no association was found between place of residence $(\mathrm{P}=0.715)$ and family history of DM $(\mathrm{P}=0.661)$ with GDM knowledge. ${ }^{9}$

In some studies, it was reported that antenatal women who were employed showed, significantly increased knowledge score when compared to housewife or unemployed women. Though in our study there was no significant difference between employed and unemployed women, knowledge about future course of GDM alone was significantly more in employed women when compared to unemployed. Probably our study population were doing household jobs and did not have any social interaction.

In our study major source of information regarding GDM awareness was from family members and friends $(69 \%, 62 \%)$. In the study by Dhyani et al. the main source of information regarding GDM awareness was from the doctors (37\%) followed by family members $(22 \%) .{ }^{9}$ In our study, health care providers were the source of information in $40.5 \%$ participants only. According to Thomas et al. and Mahalakshmi et al. the most important source of knowledge was the written/electronic media. Other sources of knowledge were the family history of diabetes and GDM, social interactions, and educational/professional experiences. ${ }^{10,14}$ Steps should be taken to make health-care workers play a greater role in creating awareness among antenatal women about GDM. Counseling about GDM, its risk factors, screening and complications during antenatal care should be included as a part of the routine health-care education programs for antenatal women io improve awareness.

\subsection{Benefits of the study}

Knowing the level of awareness of GDM among antenatal women can help us to implement different health policies to increase the same. This may play a main role in primordial prevention of GDM and thereby decrease the maternal and neonatal morbidity in future.

\subsection{Limitations of the study}

As it is a questionnaire-based study, it is subjective. Larger study population and multicentre involvement will increase the strength of the study.

\section{Conclusion}

The awareness of knowledge of GDM should be started from the school and college level by including in course curriculum. It should be more focused to people residing in rural and slum areas. Grass root level workers should be more involved in spreading awareness to all antenatal mothers from rural areas. As GDM affects two generations, knowledge about long term morbidity of GDM on mother and neonate should be emphasized to improve screening.

This study paves the path for future research to newer methods of increasing the awareness of GDM and its complications and identification of the hindrances in the acquirement of this knowledge.

\section{Declaration of competing interest}

None.

\section{Funding/support statement}

None.

\section{References}

1 Jiwani Aliya, Marseille Elliot, Lohse Nicolai, Damm Peter, Hod Moshe, James G Kahn. Gestational diabetes mellitus: results from a survey of country prevalence and practices. J Matern Fetal Neonatal Med. 2012;25(6):600-610. 
2 Seshiah V, Balaji V, Balaji MS, et al. Prevalence of gestational diabetes mellitus in South India (Tamil Nadu) — a community based study. J Assoc Phys India. 2008;56 (May):329-333.

3 Raja MW, Baba TA, Hanga AJ, et al. A study to estimate the prevalence of gestational diabetes mellites in an urban block of Kashmir valley (North India). Int J Med Sci Publ Health. 2014;3:191-195.

4 Zhu Y, Zhang C. Prevalence of gestational diabetes and risk of progression to type 2 diabetes: a global perspective. Curr Diabetes Rep. 2016;16(1):7.

5 Negrato CA, Mattar R, Gomes MB. Adverse pregnancy outcomes in women with diabetes. Diabetol Metab Syndrome. 2012. December;4(1):41.

6 Adhikari B, Mishra SR. Culture and epidemiology of diabetes in south Asia. J Glob Health. 2019;9(2), 020301.

7 Price LA, Lock LJ, Archer LE, Ahmed Z. Awareness of gestational diabetes and its risk factors among pregnant women in Samoa. Hawai'i J Med Public Health. 2017;76(2): 48-54.

8 Shriraam V, Mahadevan S, Rani S, Sathiyasekaran BWC. Awareness of gestational diabetes mellitus among antenatal women in a primary health center in South India. Indian J Endocrinol Metab. 2013;17(1):146.
9 Dhyani V, Mahantashetti NS, Ganachari MS, Kambar S, Ghatnatti V. Awareness of gestational diabetes mellitus among pregnant women attending a tertiary health center. Indian J Health Sci Biomed Res. 2018;11:51-55.

10 Mahalakshmi B, Stanly AM, Vanishree S. Awareness about gestational diabetes mellitus among antenatal women attending tertiary clinic. Int J Sci Res. 2013;2(10): $1-3$.

11 Carolan M, Steele C, Margetts H. Knowledge of gestational diabetes among a multiethnic cohort in Australia. Midwifery. 2010;26(6):579-588.

12 Bhowmik B, Afsana F, Ahmed T, et al. Evaluation of knowledge regarding gestational diabetes mellitus : a Bangladeshi study Evaluation of knowledge regarding gestational diabetes mellitus : a Bangladeshi study. Publ Health. 2018;161:67-74.

13 Bhavadharini Balaji, Mohan Deepa, Nallaperumal Sivagnanam, Mohan Anjana Ranjit, Mohan Viswanathan. Knowledge about gestational diabetes mellitus amongst pregnant women in. South Tamil Nadu. 2017;8(1):22-26.

14 Thomas Sangeetha, Pienyu Ruopfuvinuo, Santhosh Kareepadath Rajan. Awareness and knowledge about gestational diabetes mellitus among antenatal women. Psychol Community Health. 2019;8(1):237-248. 